\title{
Charged Current Quasi-Elastic Scattering at MINERvA
}

\section{Cheryl PATRICK* ${ }^{*}$}

Northwestern University

E-mail: cheryl.e.patrickegmail.com

\begin{abstract}
MINER $v$ A is a dedicated neutrino cross-section experiment running in the NuMI neutrino beam at Fermilab. We have isolated samples of both $v_{\mu}$ and $\bar{v}_{\mu}$ charged-current quasi-elastic events in the segmented scintillator tracking region of the MINERV $v$ A detector. We present the measured flux-averaged differential cross-section, $d \sigma / d Q^{2}$, compared with several models for quasielastic scattering. In both the neutrino and antineutrino cases, the data favor an axial mass $M_{A}=0.99 \mathrm{GeV} / \mathrm{c}^{2}$, but with a "transverse enhancement" modification to the nucleon form factors. This models an enhancement seen to the cross-section for electron-nucleus scattering when a transversely-polarized photon is exchanged. This model suggests nuclear effects that could lead to multiple final-state protons in the $v$ case, and an observed excess of energy around the vertex is consistent with this interpretation.
\end{abstract}

The European Physical Society Conference on High Energy Physics

18-24 July, 2013

Stockholm, Sweden

\footnotetext{
*Speaker.

${ }^{\dagger}$ On behalf of the MINER $v$ A collaboration
} 


\section{Introduction}

Two major goals of the MINER $v$ A experiment are to make high-precision quasi-elastic cross section measurements, and to identify how these are affected by the nuclear environment. In the long term, we will examine neutrino scattering cross sections on various nuclei; in this study, we look at quasi-elastic scattering from plastic scintillator, and compare our results with various nuclear models.

\section{Quasi-elastic scattering}

Quasi-elastic scattering of a neutrino (antineutrino) from a free nucleon is shown in Figure 1.

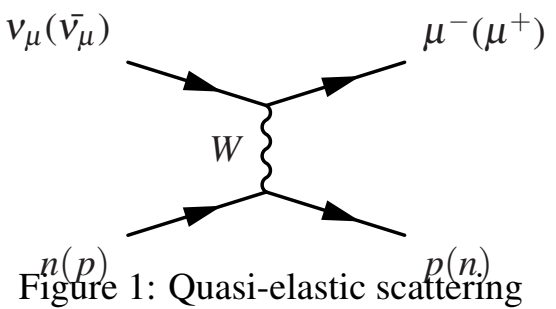

The quasi-elastic channel dominates at around $1 \mathrm{GeV}$ [3], and is thus a key signal channel for oscillation experiments, many of which operate in this energy range. It is particularly valuable because of its relatively simple kinematics; for a free nucleon, the neutrino energy $E_{V}^{Q E}$ and four-momentum transfer $Q_{Q E}^{2}$ can be reconstructed solely from the muon kinematics:

$$
\begin{gathered}
E_{v}^{Q E}=\frac{m_{n}^{2}-\left(m_{p}-E_{b}\right)^{2}-m_{\mu}^{2}+2\left(m_{p}-E_{b}\right) E_{\mu}}{2\left(m_{p}-E_{b}-E_{\mu}+p_{\mu} \cos \theta_{\mu}\right)} \\
Q_{Q E}^{2}=2 E_{v}^{Q E}\left(E_{\mu}-p_{\mu} \cos \theta_{\mu}\right)-m_{\mu}^{2}
\end{gathered}
$$

where $p_{\mu}$ and $\theta_{m u}$ represent the muon's momentum and angle (from the incoming neutrino) and $m_{p}, m_{n}$, and $m_{\mu}$ are proton, neutron and muon masses. $E_{b}$ represents a binding energy, used to generalize the quasi-elastic approximation to a nucleon contained within a nucleus.

The cross-section for quasi-elastic scattering off of a free nucleon is given by the Llewellyn Smith formalism [4]. The vector form factors involved have been well-measured by electron scattering experiments [5]. The axial form factor is typically modeled as a dipole, and has been measured with neutrino-deuterium scattering experiments, yielding an axial mass $\left(M_{A}\right)$ of 0.99 $\mathrm{GeV} / \mathrm{c}^{2}$. [6][7]. However, this distribution is modified when a nucleon is in a heavier nucleus.

\section{Nuclear effects}

Various models attempt to describe the effects of the nuclear environment on a nucleon. MINERvA's Monte Carlo generator, GENIE [9], uses a popular model of the nucleus as a relativistic Fermi gas (RFG), in which quasi-free nucleons move within a potential generated by the rest of the nucleus [8]. Additionally, final-state interactions are simulated using INTRANUKE. However, results from MiniBooNE [10][11] show deviations from this model, if the $M_{A}$ value of $0.99 \mathrm{GeV} / \mathrm{c}^{2}$ (from deuterium scattering) is used, hinting at more complex nuclear effects.

Electron scattering measurements at Jefferson Lab [12] have seen evidence of an enhancement to the electron-nucleus elastic scattering cross section when the exchanged photon is transversely 
polarized (the RFG model predicts no difference between longitudinal and transverse cross sections) (2). This effect can be parameterized as an enhancement to the magnetic form factors for the bound nucleons [15].

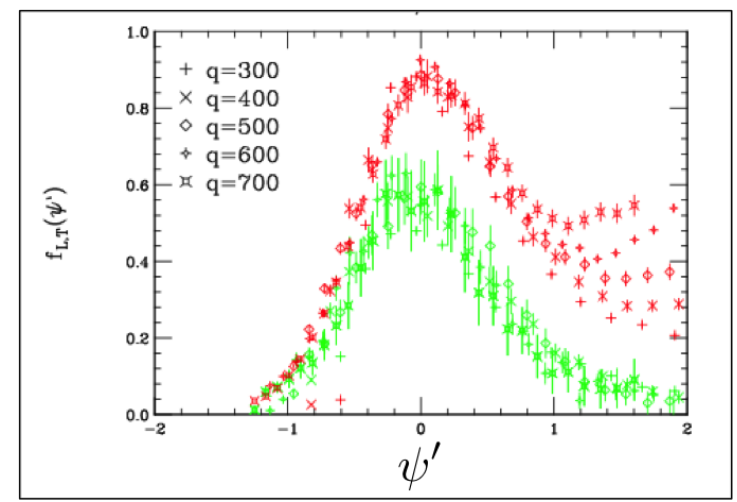

Figure 2: Transverse (red) and longitudinal (green) electron scattering cross-section vs. a scaling variable
It is believed that effects like this could be caused by correlations between initial-state nucleons. If initial nucleons do indeed form pairs, it could affect neutrino-nucleus cross section measurements, with the possible ejection of extra "partner" nucleons from the nucleus. Additionally, individual nucleons may have initial momenta above the Fermi momentum used in the relativistic Fermi gas approximation. These effects may mean that it is no longer valid to use the quasi-elastic approximation to reconstruct incoming neutrino energy.

\section{The MINER $v$ A detector}

The MINER $v$ A detector [14] sits in Fermilab's NuMI muon-neutrino beam. When collecting data for this study, the beam provided neutrinos with a mean energy around $3.5 \mathrm{GeV}$. The detector consists of 120 hexagonal modules stacked along the beam direction, surrounded by an electromagnetic calorimeter and a hadronic calorimeter. The detector comprises several nuclear targets, including water, liquid helium, iron, lead and carbon; however, this study looks at interactions in the central fully-active tracking region. This part of the detector consists of planes of interlocking triangular plastic scintillator strips oriented in three directions to create a three-dimensional track map. The MINOS near detector downstream of MINER $v$ A acts as a muon spectrometer, providing momentum and charge measurements.

\section{Quasi-elastic event selection}

MINERvA has recently published two papers showing measurements of neutrino [2] and antineutrino [1] quasi-elastic scattering on the scintillator target at energies between 1.5 and $10 \mathrm{GeV}$.

In order to isolate quasi-elastic events, we require a minimum-ionizing track that traverses the MINER $v$ A detector and enters the MINOS detector downstream, where its charge is measured and must be confirmed to correspond to a $\mu^{-}$for the neutrino case, or a $\mu^{+}$for the antineutrino. The muon track is reconstructed back to an event vertex, which must be within MINER $v$ A's fiducial volume, that is, within the central 110 planes of the tracking region, and at least $22 \mathrm{~cm}$ from all edges of the planes. Although it is expected that a neutrino-mode interaction would produce a proton in the final state, we do not require a second track. However, we impose a limit on the number of isolated energy showers, allowing a maximum of two for neutrino mode (one of these may represent a final-state proton) and one for antineutrino mode. 
Additionally, we reduce inelastic backgrounds by imposing a $Q^{2}$-dependent cut on the recoil energy. By this, we mean the sum of the calorimetric energy in a region comprising the tracker and electromagnetic calorimeter, and excluding a sphere or radius $30 \mathrm{~g} / \mathrm{cm}^{2}(v)$ or $10 \mathrm{~g} / \mathrm{cm}^{2}(\bar{v})$ around the vertex. This vertex region is large enough to contain protons up to an energy of $225 \mathrm{MeV}(v)$ or $120 \mathrm{MeV}(\bar{v})$, and is excluded from the recoil cut as this region is sensitive to low energy protons which could be emitted in quasi-elastic scattering due to initial-state nucleon correlations.

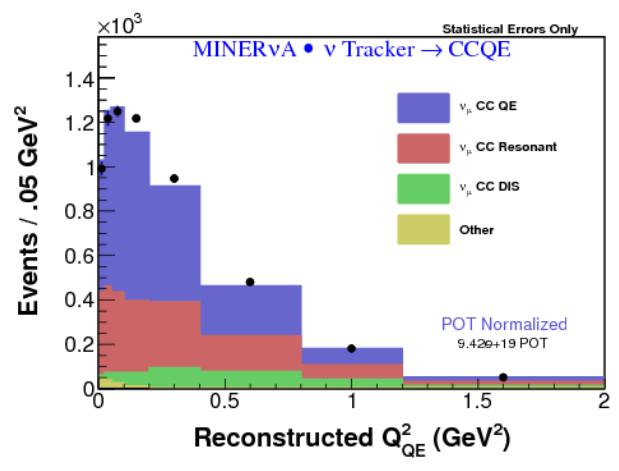

(a) Event candidates $\left(v_{\mu}\right)$

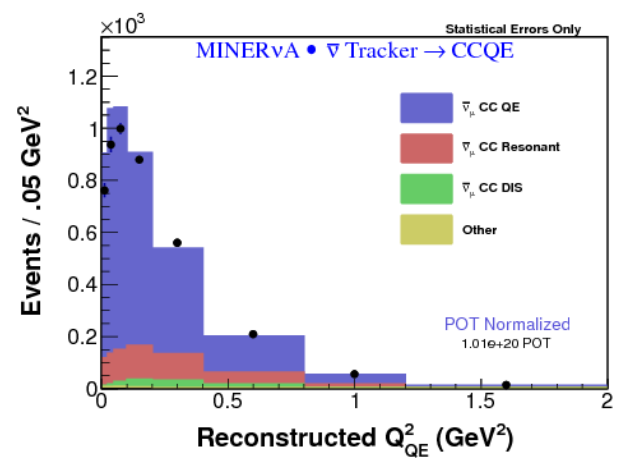

(b) Event candidates $\left(\bar{v}_{\mu}\right)$

Figure 3: Quasi-elastic event candidates in data and simulation, before background subtraction

Figure 3 shows the signal and background fractions after these cuts. In neutrino mode, we achieve $47 \%$ efficiency and $49 \%$ purity for 29,620 events, while in antineutrino mode, we have 16,467 events, with an efficiency of $54 \%$ and a purity of $77 \%$.

For each $Q_{Q E}^{2}$ bin, the background is estimated from data by taking a fit of the relative normalization of the signal and background recoil energy distributions. Once the backgrounds have been subtracted, the data is unfolded using four iterations of a Bayesian method.

Our principal source of systematic uncertainty, particularly at $Q^{2}<1 \mathrm{GeV}$, is due to uncertainty in our flux measurements (systematics are described in [2] and [1]). By area normalizing our simulations to data, we can almost eliminate the flux contribution, thus significantly reducing our overall uncertainty, such that it is dominated by statistics. The results shown will thus be in the form of area-normalized ratios to our Monte Carlo.

\section{Results and interpretation}

Figure 4 compares our data both to GENIE and to a selected models and $M_{A}$ values from the NuWro event generator [16]. It can be seen that our data disfavor the relativistic Fermi gas model with $M_{A}=0.99$ as measured in deuterium scattering. While an increase in $M_{A}$ to $1.35 \mathrm{GeV} / \mathrm{c}^{2}$ (as suggested by MiniBooNE) seems a fair fit at low $Q^{2}$ in antineutrino mode, the data diverges sharply from this model at higher $Q^{2}$ values. An addition of spectral functions to the energy-momentum relationship of the target nucleon does little to alter the RFG distribution, and does not explain our data. However, in both the neutrino and antineutrino case, our results are consistent with an $M_{A}$ of $0.99 \mathrm{GeV} / \mathrm{c}^{2}$ with the transverse enhancement model (TEM) modification to the magnetic form factors as described in [15]. This transverse enhancement is thought to be due to multi-nucleon correlations in the initial-state nucleus. 


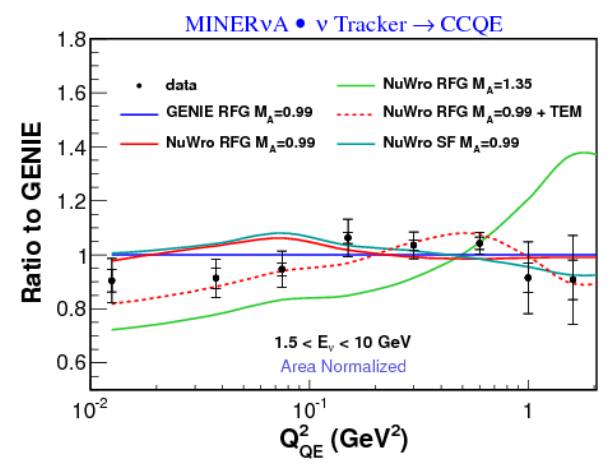

(a) Neutrino mode

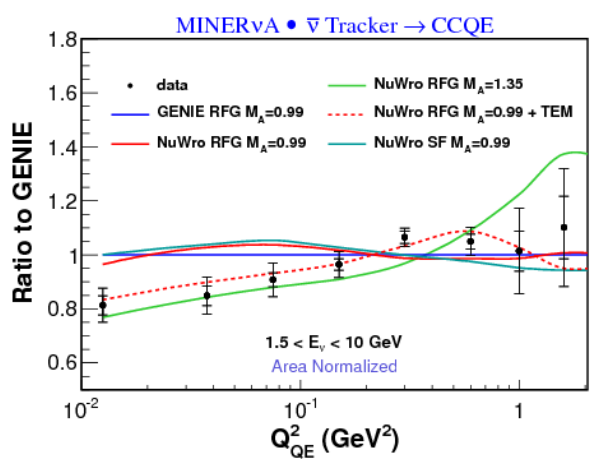

(b) Antineutrino mode

Figure 4: Comparison of cross-section results to various models

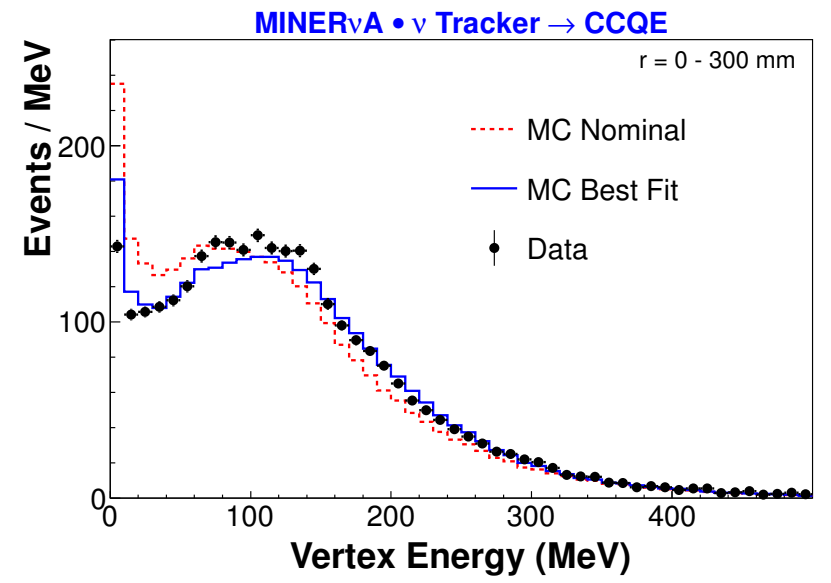

Figure 5: Vertex energy predictions, before and after the addition of extra protons
To seek evidence of multinucleon processes, we compared the energy distribution within the vertex region to simulations of additional low-energy charged particles. While no evidence of extra particles was found in antineutrino mode, the neutrino mode data favored adding an extra proton of kinetic energy below 225 $\mathrm{MeV}$ in $25 \pm 1$ (stat) \pm 9 (syst) $\%$ of cases. This could be explained by processes involving initial-state proton-neutron pairs, which could lead to interactions such as $v_{\mu}(n p) \rightarrow \mu^{-} p p$ and $\bar{v}_{\mu}(n p) \rightarrow \mu^{+} n n$. MINER $v$ A is not very sensitive to low-energy neutrons, explaining the lack of evidence of additional particles in antineutrino mode.

\section{Conclusions}

MINER $v$ A's first published cross-sections for quasi-elastic scattering on scintillator suggest that the Relativistic Fermi Gas model provides an incomplete description of nuclear processes. Our results may be explained by multi-nucleon processes, and initial studies hint at proton-neutron pairing within the nucleus. Further analyses will expand on this study by analyzing the proton track in neutrino-mode processes, and by comparing the quasi-elastic for scintillator with those in other nuclear targets, as well as looking at other processes such as inelastic scattering and pion production. 


\section{References}

[1] L. Fields, J. Chvojka et al. (MINERvA Collaboration), Measurement of Muon Antineutrino Quasi-Elastic Scattering on a Hydrocarbon Target at $E_{v} \sim 3.5 \mathrm{GeV}$, Phys. Rev. Lett. 111 (2013) 022501 [hep-ex/1305.2234].

[2] G.A. Fiorentini, D.W. Schmitz, P.A. Rodrigues et al. (MINERvA Collaboration), Measurement of Muon Neutrino Quasi-Elastic Scattering on a Hydrocarbon Target at $E_{v} \sim 3.5 \mathrm{GeV}$, Phys. Rev. Lett. 111 (2013) 022502 [hep-ex/1305.2243].

[3] A. A. Aguilar-Arevalo et al. (MiniBooNE Collaboration), Event Excess in the MiniBooNE Search for $\bar{v}_{\mu} \rightarrow \bar{v}_{\mu}$ Oscillations, Phys. Rev. Lett. 105 (2010) 081801 [hep-ex/1007.1150].

[4] C. H. Llewellyn Smith, Neutrino reactions at accelerator energies, Phys. Rept. 3 (1972) 261

[5] R. Bradford, A. Bodek, H. S. Budd, and J. Arrington, Event Excess in the MiniBooNE Search for $\bar{v}_{\mu} \rightarrow \bar{v}_{\mu}$ Oscillations, Nucl. Phys. Proc.Suppl. 159 (2006) 128 [hep-ex/ 0602017 ].

[6] A. Bodek, S. Avvakumov, R. Bradford, and H. S. Budd, Extraction of the axial nucleon form factor from neutrino experiments on deuterium, J. Phys. Conf. Ser 110 (2008) 082004 [hep-ex/0709.3538].

[7] K. S. Kuzmin, V. V. Lyubushkin, and V. A. Naumov, Quasielastic axial-vector mass from experiments on neutrinoĐnucleus scattering, Eur. Phys. J. C54 (2008) 517 [hep-ph/0712 . 4384].

[8] R. A. Smith and E. J. Moniz, Neutrino reactions on nuclear targets, Nucl. Phys. B43 (1972) 605

[9] C. Andreopoulos, A. Bell, D. Bhattacharya, F. Cavanna, J. Dobson, S. Dytman, H. Gallagher, P. Guzowski, R. Hatcher, P. Kehayias, A. Meregaglia, D. Naples, G. Pearce, A. Rubbia, M. Whalley, and T. Yang, The GENIE neutrino Monte Carlo generator, Nuclear Instruments and Methods in Physics Research Section A: Accelerators, Spectrometers, Detectors and Associated Equipment 614 (2010) 87

[10] A. A. Aguilar-Arevalo et al. (MiniBooNE Collaboration), Measurement of Muon Neutrino Quasielastic Scattering on Carbon, Phys. Rev. Lett. 100 (2008) 032301 [hep-ex/ 0706.0926 ].

[11] A. A. Aguilar-Arevalo et al. (MiniBooNE Collaboration), First Measurement of the Muon Anti-Neutrino Double-Differential Charged Current Quasi-Elastic Cross Section, Phys. Rev. D. 88 (2013) 032001 [hep-ex/1301.7067].

[12] J. Carlson, J. Jourdan, R. Schiavilla, and I. Sick, Longitudinal and transverse quasielastic response functions of light nuclei, Phys. Rev. C. 65 (2002) 024002[nucl-th/9905060].

[13] A. Bodek, H. S. Budd, and M. E. Christy, Neutrino quasi elastic scattering on nuclear targets, Eur.Phys.J. C71 (2011) 1726 [hep-ph/1106.0340].

[14] L. Aliaga et al. (MINERvA Collaboration), Design, Calibration, and Performance of the MINERvA Detector, [physics.ins-det/1305.5199].

[15] G. D'Agostini, A multidimensional unfolding method based on Bayes' theorem, Nucl.Instrum.Meth. A362 (1995) 1487

[16] T. Golan, C. Juszczak, and J. T. Sobczyk, Effects of final-state interactions in neutrino-nucleus interactions, Phys. Rev. C86 (2012) 015505 [nucl-th/1202 . 4197]. 